\title{
Neuropsychotoxicity of Abused Drugs: Potential of Dextromethorphan and Novel Neuroprotective Analogs of Dextromethorphan With Improved Safety Profiles in Terms of Abuse and Neuroprotective Effects
}

\author{
Eun-Joo Shin ${ }^{1}$, Phil Ho Lee ${ }^{2}$, Hyun Ji Kim ${ }^{1}$, Toshitaka Nabeshima ${ }^{3}$, and Hyoung-Chun Kim ${ }^{1, *}$ \\ ${ }^{I}$ Neuropsychopharmacology and Toxicology Program, College of Pharmacy, Kangwon National University, \\ Chunchon 200-701, Korea \\ ${ }^{2}$ Department of Chemistry, Kangwon National University, Chunchon, Korea \\ ${ }^{3}$ Department of Chemical Pharmacology, Meijo University Graduate School of Pharmaceutical Sciences, \\ Nagoya 468-8503, Japan
}

Received October 12, 2007; Accepted November 22, 2007

\begin{abstract}
Drug abuse involving dextromethorphan, an antitussive, has been a social problem in various geographic locations since the 1960s. Ironically, high doses of the drug confer neuroprotective activity with central nervous system and behavioral effects. Accumulating evidence suggests that metabolism to phencyclidine-like dextrorphan is not essential for the neuroprotective activity of dextromethorphan. Here, we review the neuroprotective properties of dextromethorphan and its potential for abuse and the potential neuroprotective effects of the drug's analogs and 3-hydroxymorphinan, a metabolite of dextromethorphan. These compounds may provide a novel therapeutic direction for the treatment of neurodegenerative diseases such as convulsive or parkinsonian-like disorders.
\end{abstract}

Keywords: drugs of abuse, dextromethorphan and its analog, phencyclidine-like dextrorphan, 3-hydroxymorphinan, neuroprotective effect

\section{Dextromethorphan as an antitussive}

Dextromethorphan (DM), a representative morphinan, is a centrally acting drug that increases the cough threshold, and it has been available for this indication in the United States since 1954. DM works via non-opioid receptors. Consistent with this, the opioid antagonist naloxone does not reverse the activity of DM, and codeine does not have significant activity at highaffinity DM binding sites (1). A potential advantage of $\mathrm{DM}$ is its lack of gastrointestinal side effects, such as constipation, and it causes less central nervous system (CNS) depression than opioids when used as antitussives at the usual doses. However, high doses of DM have psychotropic effects, occasionally prompting its abuse.

*Corresponding author. kimhc@kangwon.ac.kr. Published online in J-STAGE: January 16, 2008 doi: 10.1254/jphs.FM0070177

\section{Dextromethorphan abuse}

The common side effects of excessive doses of DM include a dry mouth, tachycardia, a warm feeling, and the inability to concentrate. At doses exceeding $120 \mathrm{mg}$ or about $2 \mathrm{mg} / \mathrm{kg}$ of body weight (about 10 times the therapeutic dose), DM has phencyclidine (PCP)-like effects. These include the induction of an out-of-body dreamy state, disorientation, depersonalization, confusion, somnolence or stupor, impaired coordination, agitation, distorted movement and speech, and dissociative anesthesia. Visual hallucinations are common after taking high doses $(>2.5 \mathrm{mg} / \mathrm{kg})(2-4)$; and case reports have described the recreational abuse of DM in the United States, Sweden, Australia, Germany, and Korea $(5-8)$. Abuse seems to occur primarily among adolescents and young adults, who can obtain the drug easily. Sixteen case reports of acute DM intoxication appear in the literature, although most of these ingestions were unintentional (7). In Sweden, two deaths were 


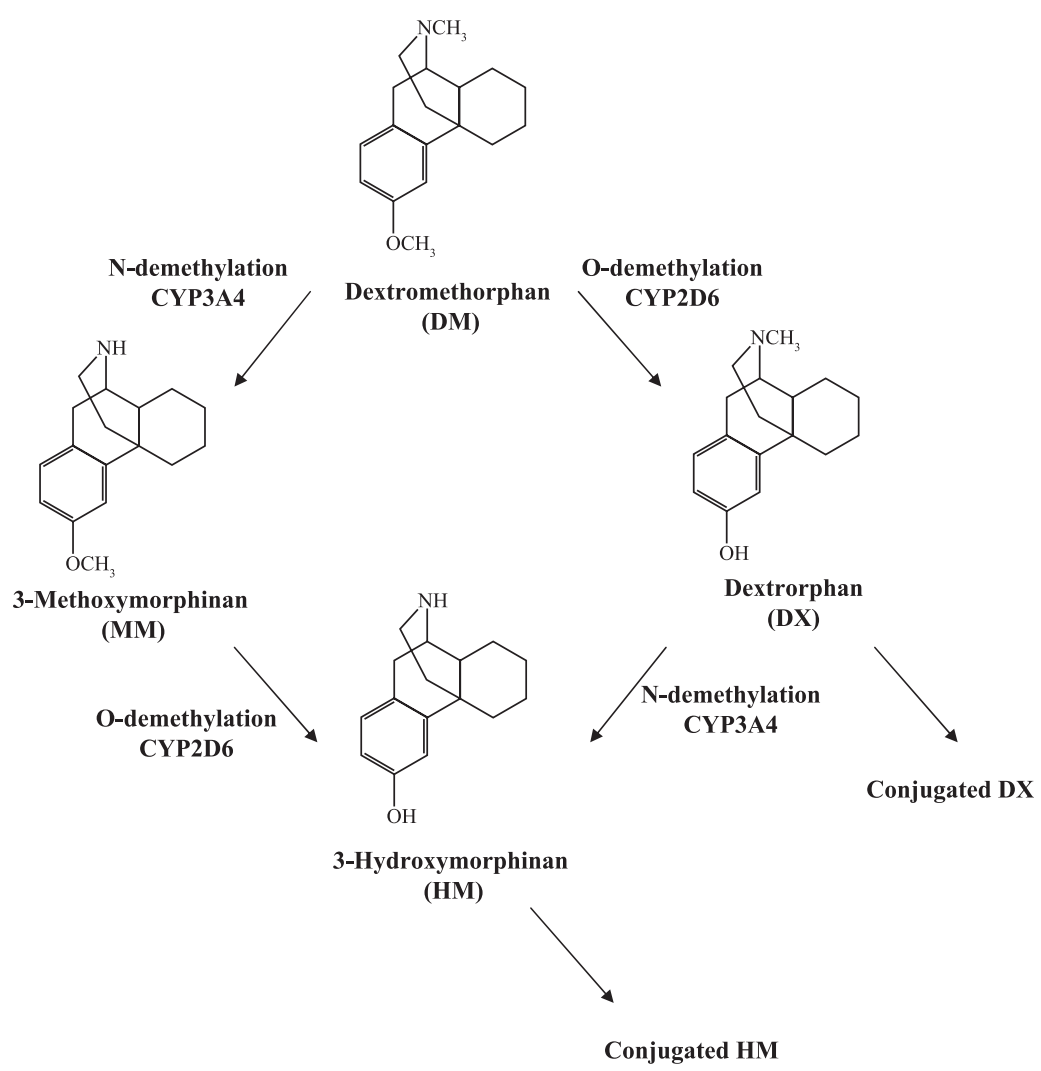

Fig. 1. Metabolic pathway of DM. CYP = cytochrome P450.

reported as a result of DM ingestion (5). With an acute overdose, the primary symptoms are related to the CNS and include altered mental status, ataxia, and nystagmus. Supportive care seems to be all that is necessary (7).

\section{Dextromethorphan-induced behavioral side effects in animals}

In mice, prolonged exposure to an oral neuroprotective dose of DM produces behavioral suppression followed by behavioral tolerance. Prenatal exposure to DM produces hyperlocomotor activity in the offspring of mice. When these offspring receive additional DM, they demonstrate marked behavioral depression (9). Mice exposed to prolonged oral DM show impaired B cell function and natural killer cell cytotoxicity, which is similar to the immunosuppressive effects of PCP (10).

As summarized in Fig. 1, DM is metabolized primarily to dextrorphan (DX) by O-demethylation and to a lesser extent, to 3-methoxymorphinan (3-MM) by $\mathrm{N}$ demethylation. Both metabolites are further demethylated to 3-hydroxymorphinan (3-HM). Urinary recovery studies in humans and rats indicate that DX and 3-HM are excreted largely ( $>95 \%)$ as glucuronide conjugates. The pharmacology of 3-MM is not significant, and it does not have major behavioral side effects. We have observed that 3-HM has antiparkinsonian effects with behavioral safety (see the section below on antiparkinsonian potential).

The major metabolite DX has neuroprotective potential (11). DX has a lower affinity than DM at the $\left[{ }^{3} \mathrm{H}\right] \mathrm{DM}$ binding site (1), but has a higher affinity than DM at the PCP receptor (12) and produces PCP-like discriminative stimulus effects (13). Therefore, the behavioral side effects of DM could be the consequence of the conversion of DM to DX. The extent of the metabolic conversion of DM to DX is highly dependent on the route of administration (14). That is, considerable DX is formed when DM is administered intraperitoneally (i.p.), whereby it is absorbed into the hepatic portal circulation, while less DX may be formed when DM is administered subcutaneously (s.c.), which avoids first-pass metabolism by the liver. However, Holtzman (15) reported that DM at $30 \mathrm{mg} / \mathrm{kg}$, s.c. has PCP-like discriminative effects of its own that are independent of the conversion to DX. These are most easily appreciated in vitro when metabolism is not an important issue. In addition, ample evidence indicates that DM has effects of its own that are independent of conversion to DX. Interestingly, DM (15) and DX (13) substituted com- 
Table 1. Pharmacological characterization of DM and its derivatives

\begin{tabular}{|c|c|c|c|c|c|c|c|}
\hline & $\mathrm{DM}$ & DX & $\mathrm{HM}$ & DF & $\begin{array}{c}\text { AM } \\
(\mathrm{CPK}-5)\end{array}$ & $\begin{array}{c}\text { CM } \\
(\mathrm{CPK}-6)\end{array}$ & AHN 649 \\
\hline DM binding site & $+++^{1}$ & $+{ }^{1}$ & $?$ & $?$ & $?$ & $?$ & $?$ \\
\hline DX binding site & + & +++ & - & - & - & - & $?$ \\
\hline Sigma 1 binding site & $+++^{32,33}$ & $+/++^{32,33}$ & $?$ & $+++^{33}$ & $+++^{32}$ & $+++^{32}$ & $++^{42}$ \\
\hline PCP-like psychotropic effect & $+{ }^{12,33}$ & $+++^{12,33}$ & -34 & -33 & -32 & -32 & $+{ }^{42}$ \\
\hline NMDA antagonistic effect & $+24,40$ & $+++^{24,40}$ & $?$ & $?$ & $?$ & $?$ & $+25,42$ \\
\hline L-type calcium channel blocking effect & $+++^{30}$ & $+^{30}$ & $?$ & $+++^{41}$ & $?$ & $?$ & ? \\
\hline Anti-inflammatory/Neurotrophic effect & $+++^{38}$ & $?$ & $+++^{34}$ & $?$ & $?$ & $?$ & $?$ \\
\hline
\end{tabular}

+++: High, ++: Moderate, +: Low, -: Negligible. Superscript numbers in the Table indicate cited reference numbers.

pletely for PCP in rats trained to distinguish PCP from saline, and DM also substituted for DX in pigeons trained to discriminate DX from saline (16). Therefore, the route of administration is an important determinant in the deposition of DM and its metabolites, as well as its behavioral effects. Other factors should also be considered in any behavioral studies of DM, including the dosage, time of testing, and capacity of glucuronidation (see Table 1).

\section{Effects of DM on the abusive drug-mediated behavioral effects}

Dextromethorphan alters the behavioral response induced by several drugs of abuse, including morphine, methamphetamine, and cocaine. DM $(25 \mathrm{mg} / \mathrm{kg}$, i.p. $)$ decreases methamphetamine self-administration in rats, but does not affect the response to a food reward (17). In addition, the coadministration of DM $(20 \mathrm{mg} / \mathrm{kg}$, i.p. $)$ with methamphetamine $(2 \mathrm{mg} / \mathrm{kg}$, i.p. $)$ attenuates the methamphetamine-induced conditioned place preference (CPP) and behavioral sensitization (18). Pretreatment with DM $(20 \mathrm{mg} / \mathrm{kg}$, s.c. $)$ potentiates the effects of acute morphine, while it attenuates the effects of chronic morphine on dopamine release in the nucleus accumbens $(17,19)$.

Pulvierenti et al. (20) demonstrated that DM $(25 \mathrm{mg}$ $/ \mathrm{kg}$, i.p.) reduces cocaine self-administration at various doses $(0.12,0.25$, and $0.5 \mathrm{mg} / \mathrm{kg}$ per $2 \mathrm{~h}$ infusion) in rats, which is in line with our previous finding (21). However, we are the first to report that DM $(40 \mathrm{mg} / \mathrm{kg}$, p.o.) increases the rate of reinforced responses to lower doses of cocaine ( 0.06 and $0.03 \mathrm{mg} / \mathrm{kg}$ per infusion) (21). Therefore, DM shifts the dose-response curve for cocaine self-administration to the left, which suggests a sensitized response to the reinforcing effects of cocaine. Consistently, DM (i.p. route) has a biphasic effect on cocaine-induced CPP. DM decreases the CPP for high doses of cocaine and increases the CPP for low doses
(22). Furthermore, we found that DM exerts biphasic effects on the locomotor stimulation induced by cocaine, and the locomotor activities parallel the Fos-related antigen immunoreactivity in the striatal complex of mice (23). Therefore, the cocaine-induced behavioral response is influenced by preexposure to DM, although the responses might be regulated by the route, period, interval of administration, or the animal model.

\section{Anti-ischemic potential}

Choi (24) reported the first evidence for a neuroprotective action of DM, and demonstrated that DM protects primary neurons exposed to glutamate from cell death. Interestingly, novel analogs of DM have been described as antagonists of glutamate-induced excitotoxic calcium channel signals in neurons. One such analog, the 3-amino-17-methylmorphinan derivative AHN649, is a safe and effective neuroprotective agent with a similar potency but improved safety profile compared to DM (25) (see Table 1, and Figs. 2 and 3).

Several in vivo models of ischemic brain injury have shown that treatment with DM protects the brain against infarction and the related pathophysiological and functional consequences of injury $(26,27)$. In particular, comprehensive studies undertaken with the rodent focal ischemia/reperfusion injury model have shown the potent ability of DM to decrease the volume of cerebral infarction and improve functional recovery following injury (25).

\section{Anticonvulsant potential}

Our studies $(28-33)$ have revealed that DM has a significant anticonvulsant effect. DM reduces the seizures and mortality and decreases the cell loss in the CA1 and CA 3 regions of the hippocampus in a dosedependent manner. DM also attenuates the kainic acid (KA)-induced increases in AP-1 DNA-binding activity 


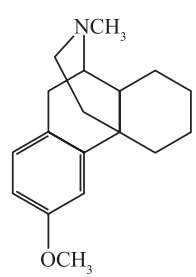

Dextromethorphan (DM)

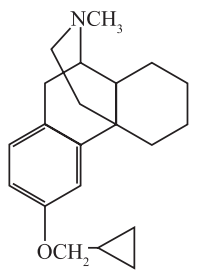

3-Cyclopropylmethoxy17-methylmorphinan ( CM )<smiles>CC1CC2CCCCC23CCCCC13</smiles>

3-Amino-

17-methylmorphinan ( AHN 649)

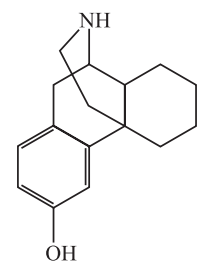

3-Hydroxymorphinan ( HM )

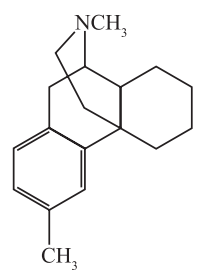

3-Methyl-N-methylmorphinan (Dimemorfan; DF )

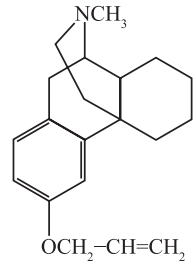

3-Allyloxy17-methylmorphinan ( AM )

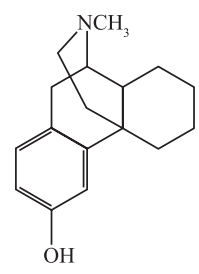

Dextrorphan (DX)
Fig. 2. Structures of DM and its analogs, including 3-allyloxy-17methylmorphinan (AM), 3-cyclopropylmethoxy-17-methylmorphinan (CM), 3-methyl- $N$-methyl-morphinan (dimemorfan: DF), the DM metabolites 3-hydroxymorphinan (3-HM) and dextrorphan (DX), and 3-amino-17-methylmorphinan (AHN 649). Based on the structure of DF or AHN 649, it cannot be metabolized to DX. and C-Jun/Fos-related antigen (FRA) expression in the hippocampus, suggesting that DM is a potent protectant against KA convulsions.

We examined the effects of a series of synthesized DM analogs (Fig. 2) that are safe behaviorally (29, $32-34$ ) and modified in positions 3 and 17 of the morphinan ring on maximal electroshock convulsions (MES) in mice (32). We found that DM, the major DM metabolite dextrorphan (DX), 3-allyloxy-17-methylmorphinan (AM), and 3-cyclopropylmethoxy-17methylmorphinan (CM) had anticonvulsant effects against MES, while other DM metabolites, such as 3methoxymorphinan (MM) and 3-hydroxymorphinan (3-HM), showed no anticonvulsant effects (32). We also observed that DM, DX, AM, and CM have high affinity to sigma 1 receptors, while they all have low affinity to sigma 2 receptors. DX has relatively higher affinity for phencyclidine (PCP) sites compared to DM. In contrast, $\mathrm{AM}$ and $\mathrm{CM}$ have very low affinities for PCP sites, suggesting that PCP sites are not required for their anticonvulsant actions $(31,32)$. Our results suggest that our DM analogs are promising anticonvulsants that lack behavioral side effects, and their anticonvulsant actions may be, at least in part, mediated via sigma 1 receptors $(32,33)$. We have also demonstrated that another DM analog, dimemorfan (DF), has a promising anticonvulsant effect with negligible behavioral side effects (33) (see Table 1 and Figs. 2 and 3).

\section{Antiparkinsonian potential}

Dextromethorphan potentiates the effects of levodopa and $\mathrm{D}_{1}$ agonists in reserpine-treated mice (35), although the results of human studies conflict. The results of an

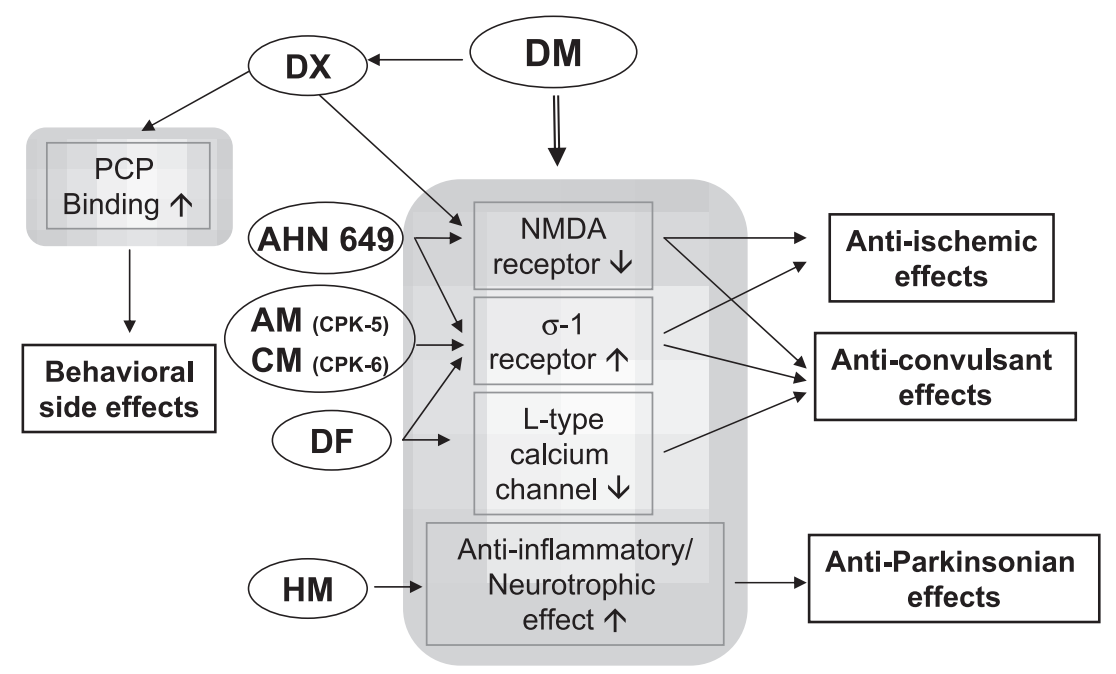

Fig. 3. Suggested neuropharmacological mechanisms of DM and its derivatives. 
open study of 11 parkinsonian patients reported by Bonucelli et al. (36) suggested that high doses (180 mg $/ \mathrm{kg}$ per day) of DM can improve some parkinsonian features (especially tremor and rigidity). In contrast, the results from Montastruc et al. (37) were disappointing. In their first series of ten patients, treatment with DM (90 mg daily) failed to relieve any PD symptoms, while in another group of eight parkinsonian patients, the drug (180 mg daily) remained either ineffective or induced major intolerable side effects (sedation, dizziness, and dysesthesia). Overall, the study did not confirm the positive results obtained by Bonucelli et al. (36).

Liu et al. (38) reported that DM protects dopaminergic (DA) neurons against inflammation-mediated degeneration. DM significantly attenuates the lipopolysaccharide (LPS)-induced reduction in the number of DA neurons. The mechanism of the neuroprotective effect of DM in the PD model may be associated with the inhibition of microglial activation, but not with its $N$-methyl D-aspartate (NMDA)-receptor antagonistic property. We also observed that 1-methyl-4-phenyl1,2,3,6-tetrahydropyridine (MPTP)-induced neuronal loss was significantly attenuated in mice receiving daily injections of DM (34).

After screening a series of DM analogs, the DM metabolite 3-HM emerged as a novel candidate for the treatment of $\mathrm{PD}(34,39)$. We demonstrated that $3-\mathrm{HM}$ is more potent in neuroprotection against the neurotoxicity induced by LPS or MPTP (34). 3-HM protects against LPS-induced DA neurotoxicity and is also neurotrophic to DA neurons in primary mixed mesencephalic neuronglia culture $(34,39)$. Therefore, 3 -HM provides potent neuroprotection by acting on two different cell targets: it has a neurotrophic effect on dopaminergic neurons and an anti-inflammatory effect on microglial activation. Compared to DM, the advantage of $3-\mathrm{HM}$ is its neurotrophic effect, which may enhance the sprouting of DA terminal fibers in the striatum. In addition, 3-HM has an anti-inflammatory effect (34) and inhibits the microgliosis generated by the damaged DA neurons induced by MPTP/MPP .

Therefore, the administration of 3-HM to MPTPinduced in vivo and in vitro PD models is beneficial for both reducing DA neuronal degeneration in the substantia nigra pars compacta $(\mathrm{SNpc})$ and restoring the depletion of biogenic amines in the striatum through its dual functions - the neurotrophic effect and reduction in reactive microgliosis - thereby providing considerable neuroprotection (see Table 1 and Figs. 2 and 3).

\section{Conclusions}

As an antitussive, DM is reported to have no CNS effects, which is an ideal characteristic. However, to achieve a neuroprotective effect, the dose of DM is much higher than the antitussive dosage. Clinically, high doses of DM induce psychotropic effects. Furthermore, $\mathrm{DM}$ is sought by drug-seeking teenagers in various geographic locales.

Our novel analogs of DM exhibit improved potency, efficacy, and safety as potential anticonvulsants, while DM exerts its neuroprotective actions with narrow beneficial effects. In particular, 3-HM, a metabolite of $\mathrm{DM}$, offers potentially potent neuroprotective action in multiple inflammatory disease models by exerting a neurotrophic effect and inhibiting the microglial activation associated with the production of a host of proinflammatory and neurotoxic factors. Therefore, these morphinans may offer a new therapeutic direction as promising compounds for the treatment of neurodegenerative disease.

\section{Acknowledgements}

This study was supported by a grant of the KoreaJapan Joint Research Program (F01-2007-000-10165-0), Korea Science and Engineering Foundation (KOSEF); by a grant (M103KV010014-07K2201-01410) from the Brain Research Center of the 21st Century Frontier Research Program funded by the Ministry of Science and Technology, Republic of Korea; by a grant of International Research Project supported by the Meijo Asian Research Center; and by a grant of the KOSEF through the National Research Laboratory Program. This program was funded by the Ministry of Science and Technology (No. M10600000203-06J0000-20310). The BK 21 program supports HJ Kim.

\section{References}

1 Craviso GL, Musacchio JM. High affinity dextromethorphan binding sites in guinea pig brain. Mol Pharmacol. 1983;23:629640 .

2 Cranston JW, Yoast R. Abuse of dextromethorphan. Arch Fam Med. 1999;8:99-100.

3 Noonan WC, Miller WR, Feeney DM. Dextromethorphan abuse among youth. Arch Fam Med. 2000;9:791-792.

4 Desai S, Aldea D, Daneels E, Soliman M, Braksmajer AS, Kopes-Kerr CP. Chronic addiction to dextromethorphan cough syrup: a case report. J Am Board Fam Med. 2006;19:320-323.

5 Rammer L, Holmgren P, Sandler H. Fatal intoxication by dextromethorphan: a report on two cases. Forensic Sci Int. 1988;37:233-236.

6 Murray S, Brewerton T. Abuse of over-the-counter dextromethorphan by teenagers. South Med J. 1993;86:1151-1153.

7 Wolfe TR, Caravati EM. Massive dextromethorphan ingestion and abuse. Am J Emerg Med. 1995;13:174-176.

8 Chung $H$. Recent trends of drug abuse and drug associated 
deaths in Korea. International Society for Neurochemistry and Japanese Forum on Nicotine and Drug Dependence Studies (Abstract). 2003. p. 72.

9 Kim HC, Jhoo WK. Alterations in motor activity induced by high dose oral administration of dextromethorphan throughout two consecutive generations in mice. Arch Pharm Res. 1995;18:146-152.

10 Kim HC, Jhoo WK, Kwon MS, Hong JS. Effects of chronic dextromethorphan administration on the cellular immune responses in mice. Arch Pharm Res. 1995;18:267-270.

11 Tortella FC, Pellicano M, Bowery NG. Dextromethorphan and neuromodulation: old drug coughs up new activities. Trends Pharmacol Sci. 1989;10:501-507.

12 Murray TF, Leid ME. Interaction of dextrorotatory opioids with phencyclidine recognotion sites in rat brain membranes. Life Sci. 1984;34:1899-1911.

13 Holtzman SG. Phencyclidine-like discriminative effects of opioids in the rats. J Pharmacol Exp Ther. 1980;214:614-619.

$14 \mathrm{Wu} \mathrm{D}$, Otton V, Kalow W, Sellers EM. Effects of route of administration on dextromethorphan pharmacokineetics and behavioural response in the rat. $\mathrm{J}$ Pharmacol Exp Ther. 1995;274:1431-1437.

15 Holtzman SG. Discriminative stimulus of dextromethorphan in the rat. Psychopharmacology. 1994;116:249-254.

16 Herling S, Solomon RE, Woods JH. Discriminative stimulus effects of dextrorphan in pigeons. J Pharamcol Exp Ther. 1983;227:723-731.

17 Glick SD, Maisonneuve IM, Dickinson HA, Kitchen BA. Comparative effects of dextromethorphan and dextrorphan on morphine, methamphetamine, and nicotine self-administration in rats. Eur J Pharmacol. 2001;422:87-90.

18 Yang PP, Huang EYK, Yeh GC, Tao PL. Co-administration of dextromethorphan with methamphetamine attenuates methamphetamine-induced rewarding and behavioural sensitization. J Biomed Sci. 2006;13:695-702.

19 Steinmiller CL, Maisonneuve IM, Glick SD. Effects of dextromethorphan on dopamine release in the nucleus accumbens: Interactions with morphine. Pharmacol Biochem Behav. 2003;74:803-810.

20 Pulvirenti L, Balducci C, Koob GF. Dextromethorphan reduces intravenous cocaine self-administration in the rat. Eur J Pharmacol. 1997;321:279-283.

21 Kim HC, Park BK, Hong SY, Jhoo WK. Dextromethorphan alters the reinforcing effect of cocaine in the rat. Meth Find Exp Clin Pharmacol. 1997; 19:627-631.

22 Jhoo WK, Shin EJ, Lee YH, Cheon MA, Oh KW, Kang SY, et al. Dual effects of dextromethorphan on cocaine-induced conditioned place preference in mice. Neurosci Lett. 2000;288:76-80.

23 Kim HC, Bing G, Shin EJ, Jhoo HS, Cheon MA, Lee SH, et al. Dextromethorphan affects cocaine-mediated behavioural pattern in parallel with a long-lasting Fos-related antigen-immunoreactivity. Life Sci. 2001;69:615-624.

24 Choi DW. Dextrorphan and dextromethorphan attenuate glutamate neurotoxicity. Brain Res. 1987;403:333-336.

25 Tortella FC, Britton P, Williams A, Lu XC, Newman AH. Neuroprotection (focal ischemia) and neurotoxicity (electroencephalographic) studies in the rats with AHN649, a 3-amino analog of dextromethorpahn and low-affinity-N-methyl-Daspartate antagonist. J Pharmacol Exp Ther. 1999;291:399-408.

26 George CP, Goldberg MP, Choi DW, Steinberg GK. Dextro- methorphan reduces neocortical ischemic neuronal damage in vivo. Brain Res. 1988;440:375-379.

27 Lo EH, Steinberg GK. Effects of dextromethorphan on regional cerebral blood flow in focal cerebral ischemia. J Cereb Blood Flow Metab. 1991;11:803-809.

28 Kim HC, Pennypacker K, Bing G, Bronstein D, McMillian M, Hong JS. The effect of dextromethorphan on kainic acid-induced seizures in the rat. Neurotoxicology. 1996;17:375-386.

29 Kim HC, Nabeshima T, Jhoo WK, Ko KH, Kim WK, Shin EJ, et al. Anticonvulsant effects of new morphinan derivatives. Bioorg Med Chem Lett. 2001;11:1651-1654.

30 Kim HC, Ko KH, Kim WK, Shin EJ, Kang KS, Shin CY, et al. Effects of dextromethorphan on the seizures induced by kainate and calcium channel antagonist BAY k-8644: comparison with the effects of dextrorphan. Behav Brain Res. 2001;120:169-175.

31 Kim HC, Bing G, Jhoo WK, Kim WK, Shin EJ, Im DH, et al. Metabolism to dextrorphan is not essential for dextromethorphan's anticonvulsant activity against kainate in mice. Life Sci. 2003;72:769-783.

32 Kim HC, Shin CY, Seo DO, Jhoo JH, Jhoo WK, Kim WK, et al. New morphinan derivatives with negligible psychotropic effects attenuate convulsions induced by maximal electroshock in mice. Life Sci. 2003;72:1883-1895.

33 Shin EJ, Nah SY, Kim WK, Ko KH, Jhoo WK, Lim YK, et al. The dextromethorphan analog dimemorfan attenuates kainateinduced seizures via $\sigma_{1}$ receptor activation: comparison with the effects of dextromethorphan. Br J Pharmacol. 2005;144:908918.

34 Zhang W, Shin EJ, Wang T, Lee PH, Pang H, Wie MB, et al. 3-Hydroxymorphinan, a metabolite of dextromethorphan, protects nigrostriatal pathway against MPTP-elicited damage both in vitro and in vivo. FASEB J. 2006;20:2496-2511.

35 Kaur S, Starr MS. Antiparkinsonian activity of dextromethorphan in the reserpine-treated mouse. Eur J Pharmacol. 1995;280:159-166.

36 Boneucelli U, Del Dotto P, Piccini P, Benge F, Corsini GU, Muratorio A. Dextromethorphan on parkinsonism. Lancet. 1992; 340:53.

37 Mantastruc JL, Fabre N, Rascol O, Senard JM, Blin O. NMethyl-D-aspartate (NMDA) antagonists and Parkinson's disease: a pilot study with dextromethorphan. Movement Disorders. 1994;9:242.

38 Liu Y, Qin L, Li G, Zhang W, An L, Liu B, et al. Dextromethorphan protects dopaminergic neurons against inflammation-mediated degeneration through inhibition of microglial activations. J Pharmacol Exp Ther. 2003;305:212-218.

39 Zhang W, Qin L, Wang T, Wei SJ, Gao HM, Liu J, et al. 3Hydroxymorphinan is neurotrophic to dopaminergic neurons and is also neuroprotective against LPS-induced neurotoxicity. FASEB J. 2005;19:395-397.

40 Choi DW, Peters S, Viseskul V. Dextrorphan and levorphanol selectively block N-methyl-D-aspartate receptor-mediated neurotoxicity on cortical neurons. J Pharmacol Exp Ther. 1987;242:713-720.

41 Shin EJ, Nabeshima T, Lee PH, Kim WK, Ko KH, Jhoo JH, et al. Dimemorfan prevents seizures induced by the L-type calcium channel activator BAYk-8644 in mice. Behav Brain Res. 2004;151:267-276.

42 Newman AH, Shah JH, Izenwasser S, Heller B, Mattson MV, Tortella FC. Highly selective sigma ligands based on dextromethorphan. Med Chem Res. 1996;6:102-117. 